\title{
Primordial planets with an admixture of dark matter particles and baryonic matter
}

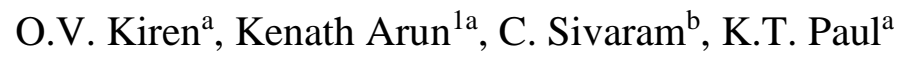

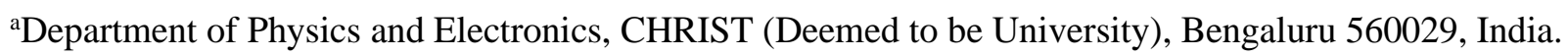 \\ ${ }^{b}$ Indian Institute of Astrophysics, Bangalore 560 034, India
}

\begin{abstract}
In our earlier work we had discussed the possibility of primordial planets composed entirely of dark matter (DM) to be the main reason for not detecting DM particles. It has been suggested that primordial planets could have formed in the early Universe and the missing baryons in the Universe could be explained by primordial free-floating planets of solid hydrogen. Many such planets were recently discovered around the old and metal poor stars and such planets could have formed at early epochs. Another possibility for missing baryons in the Universe could be that these baryons are admixed with DM particles inside the primordial planets. Here we discuss the possibility of admixture of baryons to the DM primordial planets discussed in earlier work. We consider gravitationally bound DM objects with the DM particles constituting them varying in mass from $20-100 \mathrm{GeV}$. Different fractions of DM particles mixed with baryonic matter in forming the primordial planets are discussed. For the different mass range of DM particles forming DM planets, we have estimated the radius and density of these planets with different fractions of DM and baryonic particles. It is found that for heavier mass DM particles with the admixture of certain fractions of baryonic particles, the mass of the planet increases and can reach or even substantially exceed Jupiter-mass.
\end{abstract}

Keywords: Primordial planets; Dark Matter; DM - baryonic admixture; Early Universe

\footnotetext{
${ }^{1}$ Corresponding author e-mail address: kenath.arun@christuniversity.in (Kenath Arun)
} 


\section{Introduction}

Dark matter (DM), almost five times more abundant than ordinary matter, is theorized as one of the basic constituents of the Universe. Many experiments are running worldwide to detect these DM particles but till now the interaction of these particles with the ordinary matter has proven to be so feeble that they have escaped direct detection (Arun et al., 2017). The structure formation in the Universe is a bottom-up scenario i.e. smaller objects were created first in the Universe. Sivaram and Arun, (2011), had suggested that DM particles, of several GeV rest mass, could form degenerate objects of planetary mass by gravitationally condensing. In our earlier work we had discussed the possibility of primordial planets composed entirely of DM to be the main reason for not detecting DM particles and in Sivaram et al. (2016), we had also discussed the possibility that the Neptune sized Planet Nine could be a condensed DM object.

The DM particles are heavier compared to ambient hydrogen and helium atoms and could have been accreted at earlier epochs since they are not coupled to the background cosmic radiation. In our recent paper, (Kiren et al., 2021) we had discussed the possibility of accretion of DM, Helium and Hydrogen layers on these DM objects. The evolution of these objects as the Universe expands was discussed in detail. It is not implied that all the DM particles go into forming such DM objects but the formation of such objects in large numbers in our galaxy could significantly reduce the number of free DM particles moving around in the Universe thus possibly accounting for the negative results seen in all the DM searches so far (Sivaram et al., 2019).

The typical mass of such objects made up mostly of DM particles of mass $m_{D}$, is given by (Sivaram and Arun 2011; Sivaram 1994)

$M=\frac{M_{P l}^{3}}{m_{D}^{2}}$

where the Planck mass is given by $M_{P l}=(\hbar c / G)^{1 / 2} \approx 2 \times 10^{-5} g$.

The maximum mass limit of these DM objects works out to be $10^{29} \mathrm{~g}$ (mass of Neptune), considering the mass of DM particles to be $60 \mathrm{GeV}$, favoured from the detection of excess of gamma rays from the galactic centre, attributed to the decay of $60 \mathrm{GeV} \mathrm{DM}$ particles (Huang et al. 2016; Gelmini, 2006). This mass for the DM particle is also favoured from other results (like the DAMA experiment). The radius of the DM object is given by (Sivaram and Arun, 2011):

$R=\frac{92 \hbar^{2}}{G m_{D}^{8 / 3} M^{1 / 3}}$ 
where $m_{D} \sim 60 \mathrm{GeV}$ is the DM particle mass (Huang et al., 2016).

At say $Z=10$, considering the object density to be 100 times the ambient density, the lower mass of these objects is around $10^{14} \mathrm{~g}$ (typical asteroid mass) (Sivaram et al., 2019). So the mass range of these objects varies from asteroid mass to Neptune mass.

The hypothesised Planet 9 in our solar system (Batygin and Brown, 2016; Trujillo and Sheppard, 2014), with a mass about that of Neptune, could be such a DM object (Sivaram et al. 2016). This might explain why this planet has not been visibly detected so far. It has been suggested that primordial planets could have formed in the early Universe and the missing baryons in the Universe could be explained by primordial free-floating planets of solid hydrogen. The number of such planets in the Milky Way is enormous $\sim 10^{14}$ (Wickramasinghe et al., 2012). Many such planets were recently discovered around the old and metal-poor stars and such planets could have formed at early epochs (Santos et al., 2007; Shchekinov et al., 2013; Barbato et al., 2019). Another possibility for the problem of missing baryons in the Universe could be that these baryons are admixed with DM particles inside the primordial planets.

\section{Baryonic admixture}

During the phase of formation of these primordial objects, as the primeval ambient cloud collapses we consider the presence of baryonic matter in addition to the DM particles. The number of baryonic particles present is assumed to be a fraction ' $f$ ' of the number of DM particles. If the fraction ' $f$ ' of baryonic particles is getting mixed with ' $1-f$ ' fraction of DM particles, then $m_{D}$ in equation (1) must be replaced by the effective mass of the DM-baryonic mixture given by

$m_{e f f}=\frac{(1-f) m_{D}+f m_{B}}{100}$

Thus equation (1) becomes

$M=\frac{M_{P l}^{3}}{m_{e f f}^{2}}$

When the effective mass is considered for the planetary formation, the mass of the object will increase (can be more than Jupiter mass) beyond the maximum limit $\left(10^{29} g\right.$ ) (Sivaram et al., 2016) proposed for DM planets. This happens because the effective mass $m_{\text {eff }}$ is reduced, since $m_{D} \gg m_{B}$. The corresponding radius of these planets will now be given by the formula $R=\frac{92 \hbar^{2}}{G m_{e f f}^{8 / 3} M^{1 / 3}}$ 
Here we consider the different fractions of DM particles and baryonic mixture in forming the primordial planets. For the different masses of DM particles (varying from $20 \mathrm{GeV}$ to 100 $\mathrm{GeV}$ ) forming DM planets, we have tabulated the mass, radius and density of the planets for different fractions of DM and baryonic particles using equations (3) - (5).

Considering the formation of such a planet with fraction $f=0.95$, i.e. 95\% DM particles (of mass $20 \mathrm{GeV}$ ) and $5 \%$ baryonic matter, the effective mass $m_{\text {eff }}$ works out to be $19.05 \mathrm{GeV}$. The Mass and the radius of the planet formed with this effective mass calculated from equations (4) and (5) is $8.70 \times 10^{30} \mathrm{~g}$ and $5.51 \times 10^{4} \mathrm{~cm}$. The density of the planet (which scales as $M^{2}$ ) is found to be $1.25 \times 10^{16} \mathrm{~g} / \mathrm{cm}^{3}$.

Table 1 shows the mass of the primordial planet with an admixture of DM and baryonic particles for different mass ranges of DM particles as calculated from equation (4). Table 2 shows the radius of the primordial planet with an admixture of DM and baryonic particles for different mass ranges of DM particles calculated from equation (5).

The density of these primordial planets with the admixture of DM and baryonic particles for different mass ranges of DM particles is tabulated in table 3. The mass of the planet formed for different fractions of DM and baryonic particles is plotted in Fig 1. The radius and density of these planets is plotted in Fig 2 and Fig 3.

Table 1: Mass of primordial planets with varying admixture of DM and baryonic particles (for different DM particle mass)

\begin{tabular}{|c|c|c|c|c|c|c|}
\hline \multirow{3}{*}{$\begin{array}{l}\text { Fraction } \\
f \text { of DM } \\
\text { particles (in } \\
\%)\end{array}$} & \multirow{3}{*}{$\begin{array}{l}\text { Fraction of } \\
\text { baryonic } \\
\text { particles (in } \\
\% \text { ) }\end{array}$} & \multicolumn{5}{|c|}{ Mass of DM object with baryonic admixture (g) } \\
\hline & & DM particle & DM particle & $\mathrm{DM}$ & $\mathrm{DM}$ & DM particle \\
\hline & & mass $=20$ & mass $=40$ & particle & particle & mass $=100$ \\
\hline & & $\mathrm{GeV}$ & $\mathrm{GeV}$ & mass $=60$ & mass $=80$ & $\mathrm{GeV}$ \\
\hline & & & & $\mathrm{GeV}$ & $\mathrm{GeV}$ & \\
\hline 95 & 5 & $8.70 \times 10^{30}$ & $2.18 \times 10^{30}$ & $9.70 \times 10^{29}$ & $5.46 \times 10^{29}$ & $3.49 \times 10^{29}$ \\
\hline 90 & 10 & $9.63 \times 10^{30}$ & $2.42 \times 10^{30}$ & $1.08 \times 10^{30}$ & $6.07 \times 10^{29}$ & $3.89 \times 10^{29}$ \\
\hline 85 & 15 & $1.07 \times 10^{31}$ & $2.71 \times 10^{30}$ & $1.21 \times 10^{30}$ & $6.80 \times 10^{29}$ & $4.35 \times 10^{29}$ \\
\hline 80 & 20 & $1.20 \times 10^{31}$ & $3.04 \times 10^{30}$ & $1.36 \times 10^{30}$ & $7.66 \times 10^{29}$ & $4.91 \times 10^{29}$ \\
\hline
\end{tabular}




\begin{tabular}{lllllll}
\hline $\mathbf{7 5}$ & 25 & $1.36 \times 10^{31}$ & $3.45 \times 10^{30}$ & $1.54 \times 10^{30}$ & $8.69 \times 10^{29}$ & $5.57 \times 10^{29}$ \\
\hline $\mathbf{7 0}$ & 30 & $1.54 \times 10^{31}$ & $3.94 \times 10^{30}$ & $1.76 \times 10^{30}$ & $9.96 \times 10^{29}$ & $6.39 \times 10^{29}$ \\
\hline $\mathbf{6 5}$ & 35 & $1.77 \times 10^{31}$ & $4.55 \times 10^{30}$ & $2.04 \times 10^{30}$ & $1.15 \times 10^{30}$ & $7.39 \times 10^{29}$ \\
\hline $\mathbf{6 0}$ & 40 & $2.05 \times 10^{31}$ & $5.30 \times 10^{30}$ & $2.38 \times 10^{30}$ & $1.35 \times 10^{30}$ & $8.65 \times 10^{29}$ \\
\hline $\mathbf{5 5}$ & 45 & $2.41 \times 10^{31}$ & $6.26 \times 10^{30}$ & $2.82 \times 10^{30}$ & $1.60 \times 10^{30}$ & $1.03 \times 10^{30}$ \\
\hline $\mathbf{5 0}$ & 50 & $2.86 \times 10^{31}$ & $7.51 \times 10^{30}$ & $3.39 \times 10^{30}$ & $1.92 \times 10^{30}$ & $1.24 \times 10^{30}$ \\
\hline $\mathbf{4 5}$ & 55 & $3.46 \times 10^{31}$ & $9.17 \times 10^{30}$ & $4.16 \times 10^{30}$ & $2.36 \times 10^{30}$ & $1.52 \times 10^{30}$ \\
\hline $\mathbf{4 0}$ & 60 & $4.27 \times 10^{31}$ & $1.15 \times 10^{31}$ & $5.22 \times 10^{30}$ & $2.97 \times 10^{30}$ & $1.91 \times 10^{30}$ \\
\hline $\mathbf{3 5}$ & 65 & $5.39 \times 10^{31}$ & $1.47 \times 10^{31}$ & $6.73 \times 10^{30}$ & $3.85 \times 10^{30}$ & $2.48 \times 10^{30}$ \\
\hline $\mathbf{3 0}$ & 70 & $7.03 \times 10^{31}$ & $1.96 \times 10^{31}$ & $9.03 \times 10^{30}$ & $5.17 \times 10^{30}$ & $3.35 \times 10^{30}$ \\
\hline $\mathbf{2 5}$ & 75 & $9.55 \times 10^{31}$ & $2.73 \times 10^{31}$ & $1.27 \times 10^{31}$ & $7.33 \times 10^{30}$ & $4.76 \times 10^{30}$ \\
\hline $\mathbf{2 0}$ & 80 & $1.37 \times 10^{32}$ & $4.08 \times 10^{31}$ & $1.93 \times 10^{31}$ & $1.12 \times 10^{31}$ & $7.30 \times 10^{30}$ \\
\hline $\mathbf{1 5}$ & 85 & $2.13 \times 10^{32}$ & $6.73 \times 10^{31}$ & $3.25 \times 10^{31}$ & $1.91 \times 10^{31}$ & $1.26 \times 10^{31}$ \\
\hline $\mathbf{1 0}$ & 90 & $3.75 \times 10^{32}$ & $1.31 \times 10^{32}$ & $6.63 \times 10^{31}$ & $3.98 \times 10^{31}$ & $2.66 \times 10^{31}$ \\
\hline $\mathbf{5}$ & 95 & $8.30 \times 10^{32}$ & $3.63 \times 10^{32}$ & $2.02 \times 10^{32}$ & $1.29 \times 10^{32}$ & $8.92 \times 10^{31}$ \\
\hline & & & & & & \\
\hline & & & & & & \\
\hline
\end{tabular}

Table 2: Radius of primordial planets with varying admixture of DM and baryonic particles (for different DM particle mass)

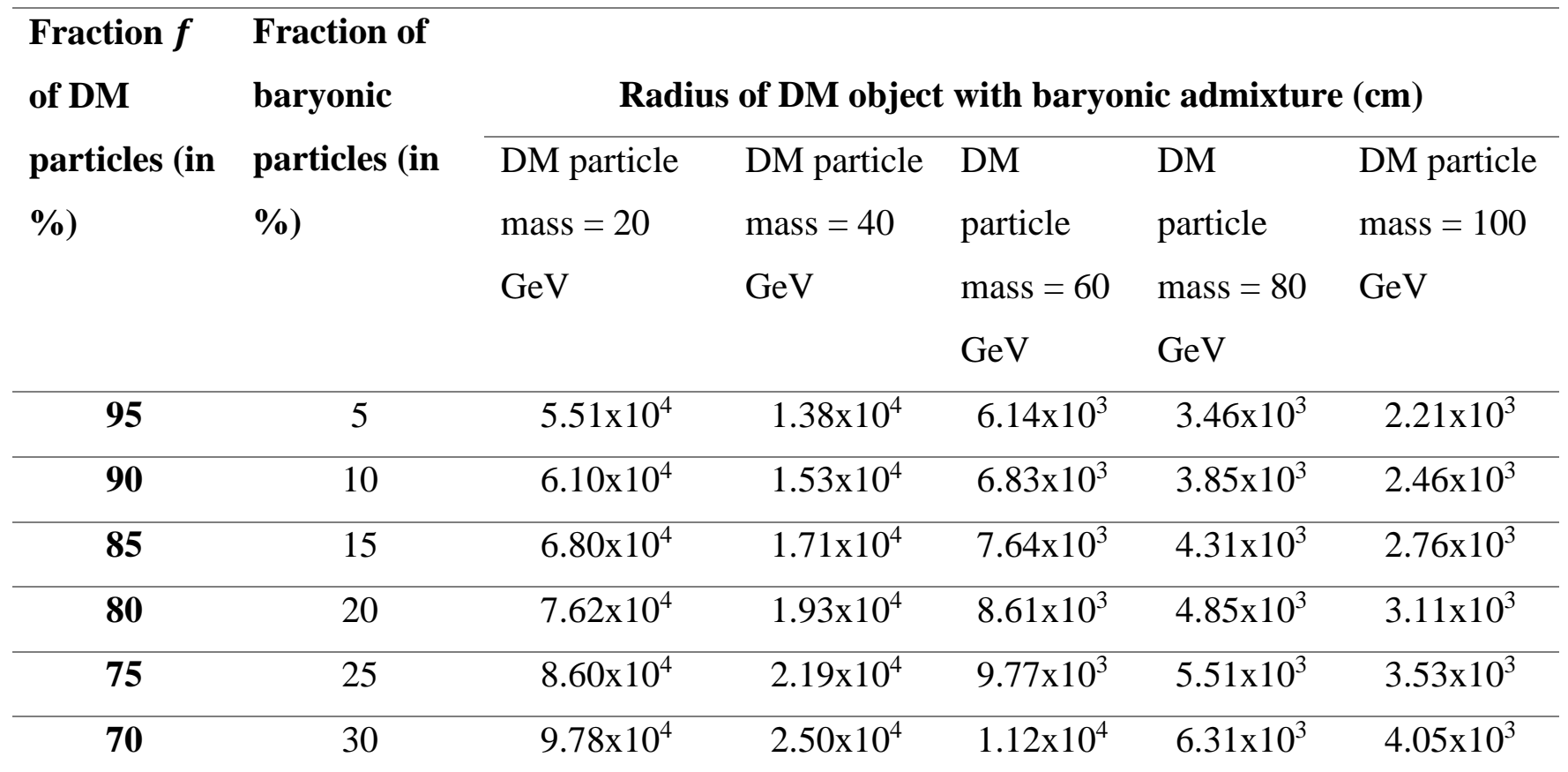




\begin{tabular}{ccccccc}
\hline $\mathbf{6 5}$ & 35 & $1.12 \times 10^{5}$ & $2.88 \times 10^{4}$ & $1.29 \times 10^{4}$ & $7.30 \times 10^{3}$ & $4.68 \times 10^{3}$ \\
\hline $\mathbf{6 0}$ & 40 & $1.30 \times 10^{5}$ & $3.36 \times 10^{4}$ & $1.51 \times 10^{4}$ & $8.54 \times 10^{3}$ & $5.48 \times 10^{3}$ \\
\hline $\mathbf{5 5}$ & 45 & $1.53 \times 10^{5}$ & $3.97 \times 10^{4}$ & $1.79 \times 10^{4}$ & $1.01 \times 10^{4}$ & $6.50 \times 10^{3}$ \\
\hline $\mathbf{5 0}$ & 50 & $1.81 \times 10^{5}$ & $4.76 \times 10^{4}$ & $2.15 \times 10^{4}$ & $1.22 \times 10^{4}$ & $7.84 \times 10^{3}$ \\
\hline $\mathbf{4 5}$ & 55 & $2.19 \times 10^{5}$ & $5.81 \times 10^{4}$ & $2.63 \times 10^{4}$ & $1.50 \times 10^{4}$ & $9.64 \times 10^{3}$ \\
\hline $\mathbf{4 0}$ & 60 & $2.70 \times 10^{5}$ & $7.26 \times 10^{4}$ & $3.30 \times 10^{4}$ & $1.88 \times 10^{4}$ & $1.21 \times 10^{4}$ \\
\hline $\mathbf{3 5}$ & 65 & $3.42 \times 10^{5}$ & $9.32 \times 10^{4}$ & $4.27 \times 10^{4}$ & $2.44 \times 10^{4}$ & $1.57 \times 10^{4}$ \\
\hline $\mathbf{3 0}$ & 70 & $4.46 \times 10^{5}$ & $1.24 \times 10^{5}$ & $5.72 \times 10^{4}$ & $3.28 \times 10^{4}$ & $2.12 \times 10^{4}$ \\
\hline $\mathbf{2 5}$ & 75 & $6.05 \times 10^{5}$ & $1.73 \times 10^{5}$ & $8.06 \times 10^{4}$ & $4.64 \times 10^{4}$ & $3.02 \times 10^{4}$ \\
\hline $\mathbf{2 0}$ & 80 & $8.68 \times 10^{5}$ & $2.58 \times 10^{5}$ & $1.22 \times 10^{5}$ & $7.09 \times 10^{4}$ & $4.62 \times 10^{4}$ \\
\hline $\mathbf{1 5}$ & 85 & $1.35 \times 10^{6}$ & $4.26 \times 10^{5}$ & $2.06 \times 10^{5}$ & $1.21 \times 10^{5}$ & $7.96 \times 10^{4}$ \\
\hline $\mathbf{1 0}$ & 90 & $2.38 \times 10^{6}$ & $8.33 \times 10^{5}$ & $4.20 \times 10^{5}$ & $2.52 \times 10^{5}$ & $1.68 \times 10^{5}$ \\
\hline $\mathbf{5}$ & 95 & $5.26 \times 10^{6}$ & $2.30 \times 10^{6}$ & $1.28 \times 10^{6}$ & $8.16 \times 10^{5}$ & $5.65 \times 10^{5}$
\end{tabular}

Table 3: Density of primordial planets with varying admixture of DM and baryonic particles (for different DM particle mass)

\begin{tabular}{|c|c|c|c|c|c|c|}
\hline \multirow{5}{*}{$\begin{array}{l}\text { Fraction } \boldsymbol{f} \\
\text { of DM } \\
\text { particles (in } \\
\%)\end{array}$} & \multirow{3}{*}{$\begin{array}{l}\text { Fraction of } \\
\text { baryonic } \\
\text { particles (in } \\
\% \text { ) }\end{array}$} & \multicolumn{5}{|c|}{ Density of DM object with baryonic admixture $\left(\mathrm{g} / \mathrm{cm}^{3}\right)$} \\
\hline & & DM particle & DM particle & $\mathrm{DM}$ & $\mathrm{DM}$ & DM particle \\
\hline & & mass $=20$ & mass $=40$ & particle & particle & $\operatorname{mass}=100$ \\
\hline & & $\mathrm{GeV}$ & $\mathrm{GeV}$ & mass $=60$ & mass $=80$ & $\mathrm{GeV}$ \\
\hline & & & & $\mathrm{GeV}$ & $\mathrm{GeV}$ & \\
\hline 95 & 5 & $1.25 \times 10^{16}$ & $1.99 \times 10^{17}$ & $1.00 \times 10^{18}$ & $3.17 \times 10^{18}$ & $7.73 \times 10^{18}$ \\
\hline 90 & 10 & $1.02 \times 10^{16}$ & $1.61 \times 10^{17}$ & $8.11 \times 10^{17}$ & $2.56 \times 10^{18}$ & $6.24 \times 10^{18}$ \\
\hline 85 & 15 & $8.19 \times 10^{15}$ & $1.29 \times 10^{17}$ & $6.48 \times 10^{17}$ & $2.04 \times 10^{18}$ & $4.98 \times 10^{18}$ \\
\hline 80 & 20 & $6.52 \times 10^{15}$ & $1.02 \times 10^{17}$ & $5.11 \times 10^{17}$ & $1.61 \times 10^{18}$ & $3.92 \times 10^{18}$ \\
\hline 75 & 25 & $5.12 \times 10^{15}$ & $7.93 \times 10^{16}$ & $3.97 \times 10^{17}$ & $1.25 \times 10^{18}$ & $3.04 \times 10^{18}$ \\
\hline 70 & 30 & $3.96 \times 10^{15}$ & $6.07 \times 10^{16}$ & $3.03 \times 10^{17}$ & $9.51 \times 10^{17}$ & $2.31 \times 10^{18}$ \\
\hline 65 & 35 & $3.01 \times 10^{15}$ & $4.57 \times 10^{16}$ & $2.27 \times 10^{17}$ & $7.11 \times 10^{17}$ & $1.73 \times 10^{18}$ \\
\hline 60 & 40 & $2.24 \times 10^{15}$ & $3.36 \times 10^{16}$ & $1.66 \times 10^{17}$ & $5.20 \times 10^{17}$ & $1.26 \times 10^{18}$ \\
\hline
\end{tabular}




\begin{tabular}{lllllll}
\hline $\mathbf{5 5}$ & 45 & $1.63 \times 10^{15}$ & $2.41 \times 10^{16}$ & $1.19 \times 10^{17}$ & $3.70 \times 10^{17}$ & $8.95 \times 10^{17}$ \\
\hline $\mathbf{5 0}$ & 50 & $1.15 \times 10^{15}$ & $1.67 \times 10^{16}$ & $8.20 \times 10^{16}$ & $2.55 \times 10^{17}$ & $6.16 \times 10^{17}$ \\
\hline $\mathbf{4 5}$ & 55 & $7.88 \times 10^{14}$ & $1.12 \times 10^{16}$ & $5.46 \times 10^{16}$ & $1.69 \times 10^{17}$ & $4.08 \times 10^{17}$ \\
\hline $\mathbf{4 0}$ & 60 & $5.18 \times 10^{14}$ & $7.19 \times 10^{15}$ & $3.47 \times 10^{16}$ & $1.07 \times 10^{17}$ & $2.57 \times 10^{17}$ \\
\hline $\mathbf{3 5}$ & 65 & $3.24 \times 10^{14}$ & $4.36 \times 10^{15}$ & $2.08 \times 10^{16}$ & $6.38 \times 10^{16}$ & $1.53 \times 10^{17}$ \\
\hline $\mathbf{3 0}$ & 70 & $1.91 \times 10^{14}$ & $2.46 \times 10^{15}$ & $1.16 \times 10^{16}$ & $3.52 \times 10^{16}$ & $8.41 \times 10^{16}$ \\
\hline $\mathbf{2 5}$ & 75 & $1.04 \times 10^{14}$ & $1.26 \times 10^{15}$ & $5.83 \times 10^{15}$ & $1.76 \times 10^{16}$ & $4.16 \times 10^{16}$ \\
\hline $\mathbf{2 0}$ & 80 & $5.03 \times 10^{13}$ & $5.68 \times 10^{14}$ & $2.54 \times 10^{15}$ & $7.54 \times 10^{15}$ & $1.77 \times 10^{16}$ \\
\hline $\mathbf{1 5}$ & 85 & $2.08 \times 10^{13}$ & $2.09 \times 10^{14}$ & $8.91 \times 10^{14}$ & $2.58 \times 10^{15}$ & $5.98 \times 10^{15}$ \\
\hline $\mathbf{1 0}$ & 90 & $6.70 \times 10^{12}$ & $5.46 \times 10^{13}$ & $2.15 \times 10^{14}$ & $5.94 \times 10^{14}$ & $1.34 \times 10^{15}$ \\
\hline $\mathbf{5}$ & 95 & $1.37 \times 10^{12}$ & $7.17 \times 10^{12}$ & $2.31 \times 10^{13}$ & $5.69 \times 10^{13}$ & $1.19 \times 10^{14}$ \\
\hline
\end{tabular}
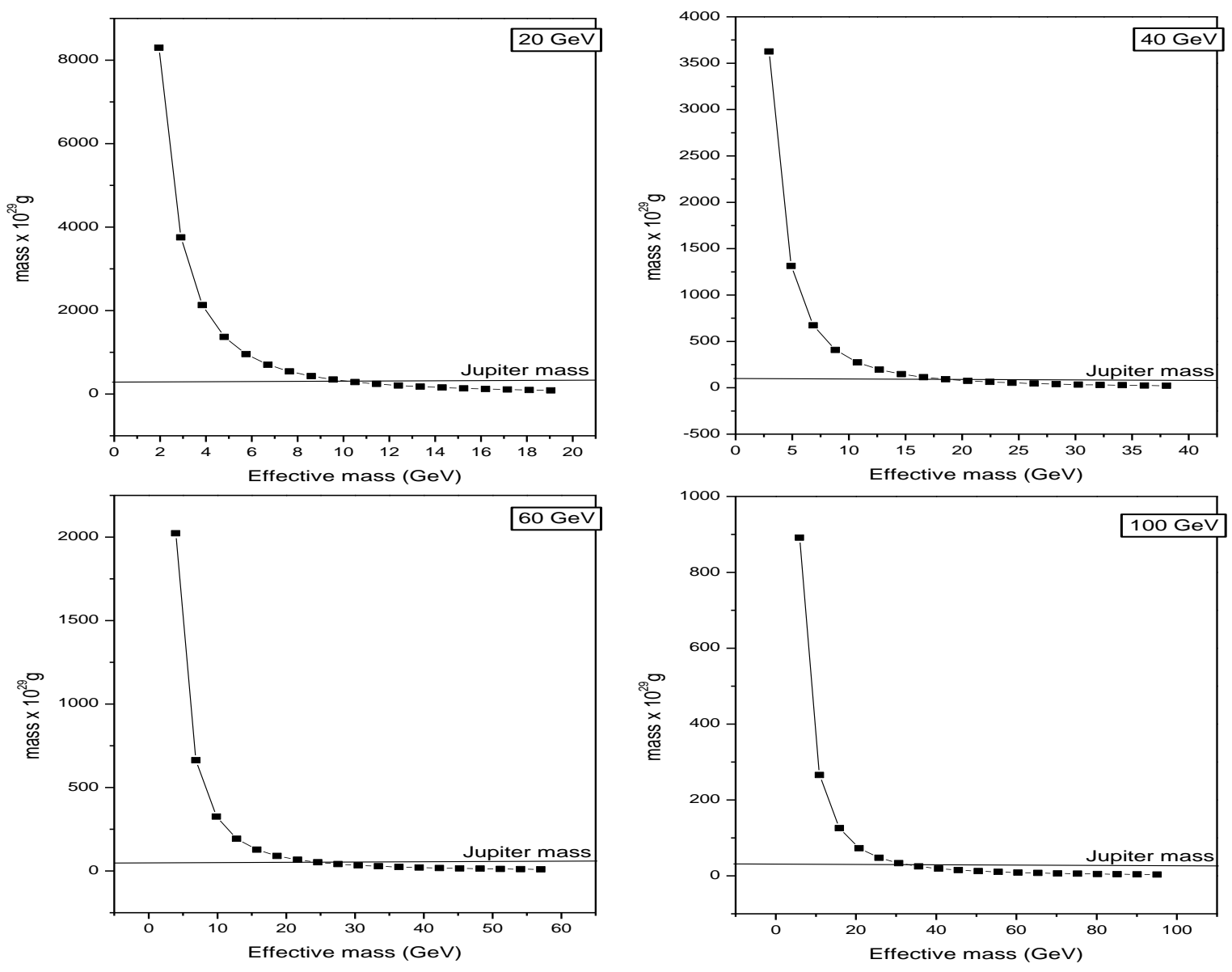

Figure 1: Mass of DM planet admixed with baryonic matter for different DM particle mass 

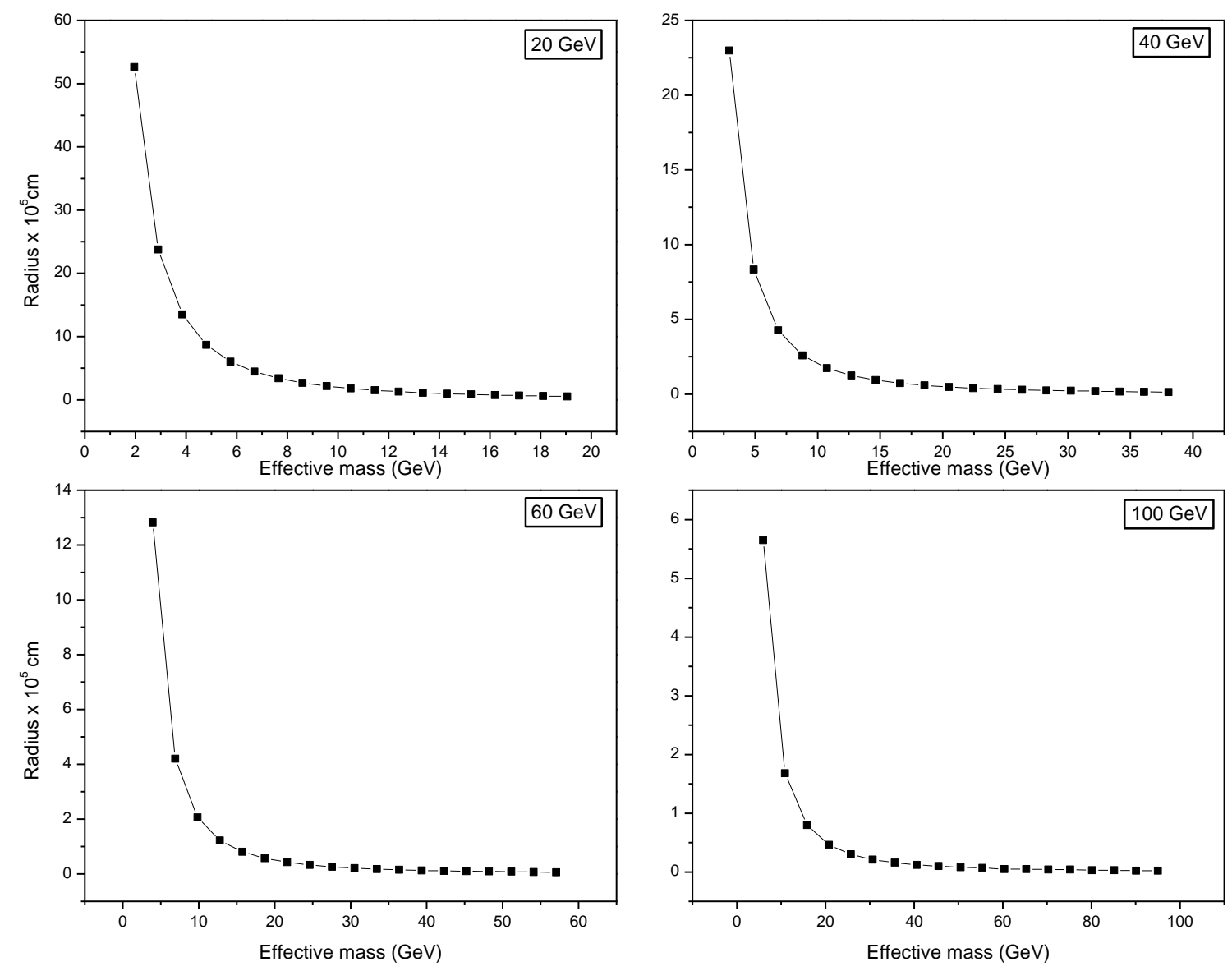

Figure 2: Radius of DM planet admixed with baryonic matter for different DM particle mass

Table 1 shows that the mass of DM planets with an admixture of baryonic particles increases its mass limit beyond the Neptune mass. For planets made entirely of DM, the maximum mass limit was Neptune mass and it can go down to asteroid mass. But with an admixture of DM and baryonic particles, the mass of the planet increases with the increase in the fraction of baryonic matter. When the baryonic fraction to that of DM particle increases, the planetary mass can increase and go beyond the Jupiter mass (up to 40 times Jupiter mass, as given in Table 1 and Fig 1).

For $20 \mathrm{GeV}$ and $40 \mathrm{GeV}$ DM particle admixed with baryonic particles, the planets formed have masses beyond Jupiter mass. For $60 \mathrm{GeV}$ DM particle, admixed with baryonic particles, planets formed with $f<0.65$ will have mass beyond Jupiter mass. For $80 \mathrm{GeV}$ and $100 \mathrm{GeV} \mathrm{DM}$ 
particles, admixed with baryonic particles, planets formed with $f<0.5$ and $f<0.4$ will have mass beyond Jupiter mass. Since a large fraction of exoplanets observed have masses well beyond the Jupiter mass, some of the exoplanets could be these objects. Also from table 1, it is found that some objects can have sub stellar masses (about 50 Jupiter-mass), like that of brown dwarfs.
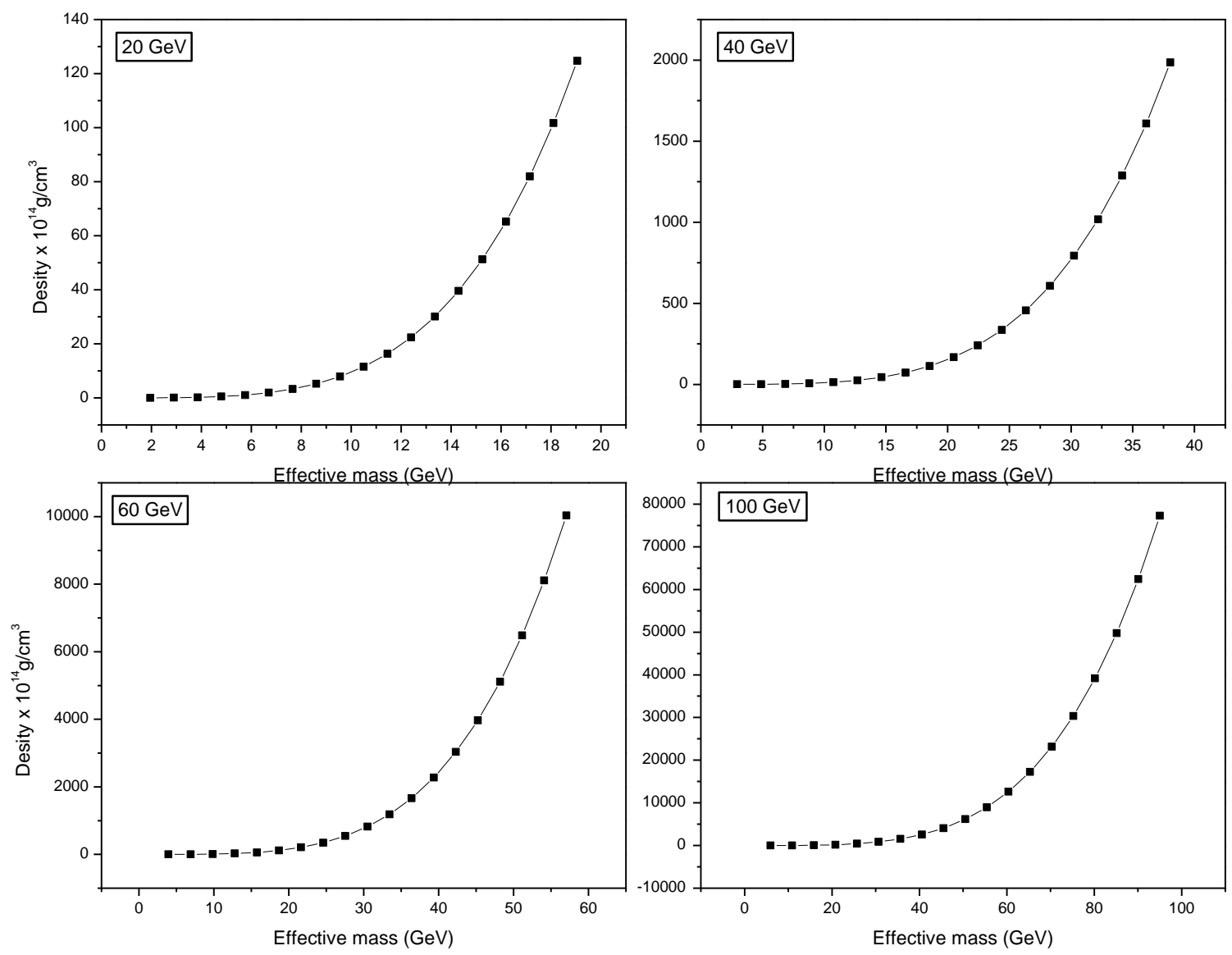

Figure 3: Density of DM planet admixed with the baryonic matter for different DM particle mass

The radius of these planets (admixed with DM and baryonic matter) also increases with an increase in the fraction of baryonic matter. But it is found from Table 2 that if the DM particles involved in the formation of these planets are heavier, the size of planets is reduced (by an order of 10) compared to the planets being formed by lighter DM particles. The density of these planets on the other hand decreases with an increase in the fraction of baryonic matter. Density is higher for planets formed with heavier DM particles than planets formed with lighter DM particles. 


\section{Conclusions}

The mass of primordial planets (that has admixture of both DM and baryonic particles) will increase with the fraction of baryonic matter in the planet and its mass can go well beyond the mass of Jupiter (around 40 times Jupiter mass) and can also approach sub-stellar mass (brown dwarf mass). So far, thousands of exoplanets have been discovered by the Kepler mission and more will be found by NASA's Transiting Exoplanet Survey Satellite (TESS) mission, which is observing the entire sky to locate planets orbiting the nearest and brightest stars.

Many exoplanets (exo-Jupiters) discovered so far fall in this mass range and we are not very sure whether these exoplanets are entirely made of baryons. Some of the exoplanets with mass several times Jupiter mass could be possible signatures of the presence of primordial planets with an admixture of baryonic and DM particles. It is also found that some of these planets could reach even sub stellar mass $\left(10^{32} \mathrm{~g}\right)$ like that of brown dwarf (Kirkpatrick et al., 2021).

Also, even if a small fraction of DM particles is trapped in these objects, the flux of ambient DM particles would be reduced significantly. This could be one of the many reasons for not detecting the DM particles in various experiments like XENON1T experiment, ADMX etc. as suggested earlier (Kiren et al., 2021).

\section{References}

- Arun, K., Gudennavar, S.B. and Sivaram, C., 2017. Dark matter, dark energy, and alternate models: A review. Adv. Space Res. 60, 166.

- Barbato, D. et al., 2019. The GAPS Programme with HARPS-N at TNG-XVIII. Two new giant planets around the metal-poor stars HD 220197 and HD 233832. Astron. Astrophys. 621, A110.

- Batygin, K., Brown, M.E., 2016. Evidence for a distant giant planet in the solar system. Astron. J. 151, 22.

- Gelmini, G.B., 2006. DAMA detection claim is still compatible with all other DM searches. J. Phys. Conf. Ser. 39, 166.

- Huang, X.J., Zhang, W.H., Zhou, Y.F., 2016. $750 \mathrm{GeV}$ diphoton excess and a dark matter messenger at the Galactic Center. Phys. Rev. D 93, 115006.

- Kiren, O.V., Arun, K., Sivaram, C., 2021. Evolution of primordial dark matter planets in the early Universe. Adv. Space Res. 68, 2050. 
- Kirkpatrick, J.D. et al., 2021. The Enigmatic Brown Dwarf WISEA J153429. 75-104303.3 (aka “The Accident”). Astrophys. J. Lett. 915, L6.

- Santos, N.C. et al., 2007. The HARPS search for southern extra-solar planets-XII. A giant planet orbiting the metal-poor star HD 171028. Astron. Astrophys. 474, 647.

- Shchekinov, Y.A., Safonova, M., Murthy, J., 2013. Planets in the early Universe. Astrophys. Space Sci. 346, 31.

- Sivaram, C., 1994. Mond, dark matter and the cosmological constant. Astrophys. Space Sci. 219, 135.

- Sivaram, C., Arun, K. 2011. New class of dark matter objects and their detection. Open Astron. J. 4, 57.

- Sivaram, C., Arun, K., Kiren, O.V., 2016. Planet Nine, dark matter and MOND. Astrophys. Space Sci. 361, 1.

- Sivaram, C., Arun, K., Kiren, O.V., 2019. Primordial planets predominantly of dark matter. Earth Moon Planets 122, 115.

- Trujillo, C.A., Sheppard, S.S., 2014. A Sedna-like body with a perihelion of 80 astronomical units. Nature 507, 471.

- Wickramasinghe, N.C. et al., 2012. Life-bearing primordial planets in the solar vicinity. Astrophys. Space Sci. 341, 295. 